\title{
GeNNet: an integrated platform for unifying scientific workflows and graph databases for transcriptome data analysis
}

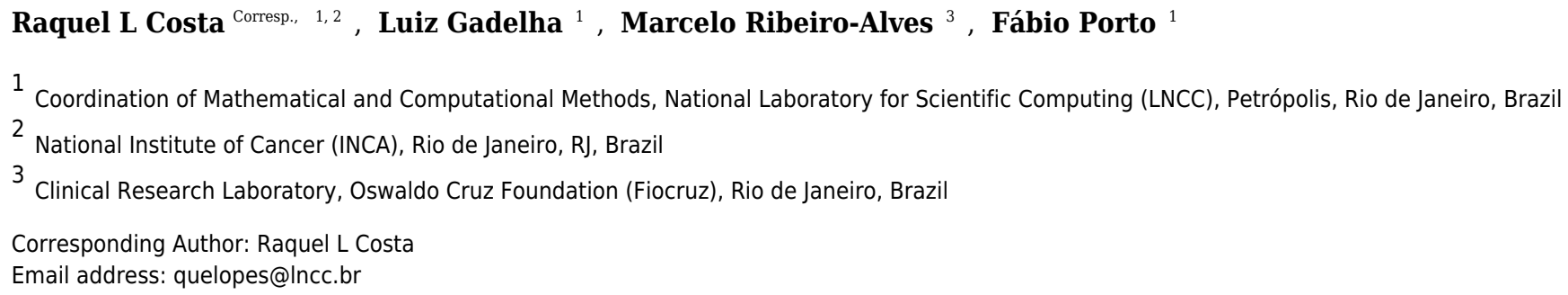

There are many steps in analyzing transcriptome data, from the acquisition of raw data to the selection of a subset of representative genes that explain a scientific hypothesis. The data produced can be represented as networks of interactions among genes and these may additionally be integrated with other biological databases, such as Protein-Protein Interactions, transcription factors and gene annotation. However, the results of these analyses remain fragmented, imposing difficulties, either for posterior inspection of results, or for meta-analysis by the incorporation of new related data. Integrating databases and tools into scientific workflows, orchestrating their execution, and managing the resulting data and its respective metadata are challenging tasks. Additionally, a great amount of effort is equally required to run in-silico experiments to structure and compose the information as needed for analysis. Different programs may need to be applied and different files are produced during the experiment cycle. In this context, the availability of a platform supporting experiment execution is paramount. We present GeNNet, an integrated transcriptome analysis platform that unifies scientific workflows with graph databases for selecting relevant genes according to the evaluated biological systems. It includes GeNNet-Wf, a scientific workflow that pre-loads biological data, pre-processes raw microarray data and conducts a series of analyses including normalization, differential expression inference, clusterization and gene set enrichment analysis. A user-friendly web interface, GeNNet-Web, allows for setting parameters, executing, and visualizing the results of GeNNet-Wf executions. To demonstrate the features of GeNNet, we performed case studies with data retrieved from GEO, particularly using a single-factor experiment in different analysis scenarios. As a result, we obtained differentially expressed genes for which biological functions were analyzed. The results are integrated into GeNNet-DB, a database about genes, clusters, experiments and their properties and relationships. The 
resulting graph database is explored with queries that demonstrate the expressiveness of this data model for reasoning about gene interaction networks. GeNNet is the first platform to integrate the analytical process of transcriptome data with graph databases. It provides a comprehensive set of tools that would otherwise be challenging for non-expert users to install and use. Developers can add new functionality to components of GeNNet. The derived data allows for testing previous hypotheses about an experiment and exploring new ones through the interactive graph database environment. It enables the analysis of different data on humans, rhesus, mice and rat coming from Affymetrix platforms. GeNNet is available as an open source platform at https://github.com/raquele/GeNNet and can be retrieved as software container with the command docker pull quelopes/gennet. 


\title{
GeNNet: an integrated platform for unifying scientific workflows and graph databases for transcriptome data analysis
}

\author{
Raquel L. Costa ${ }^{1,2}$, Luiz M. R. Gadelha Jr. ${ }^{1}$, Marcelo Ribeiro-Alves ${ }^{3}$, and Fábio \\ Porto ${ }^{1}$
}

\author{
${ }^{1}$ National Laboratory for Scientific Computing (LNCC), Petrópolis, Brazil \\ ${ }^{2}$ National Institute of Cancer (INCA), Rio de Janeiro, Brazil \\ ${ }^{3}$ Oswaldo Cruz Foundation (Fiocruz), Rio de Janeiro, Brazil \\ Corresponding author: \\ First Author ${ }^{1}$ \\ Email address: quelopes@Incc.br
}

\begin{abstract}
There are many steps in analyzing transcriptome data, from the acquisition of raw data to the selection of a subset of representative genes that explain a scientific hypothesis. The data produced can be represented as networks of interactions among genes and these may additionally be integrated with other biological databases, such as Protein-Protein Interactions, transcription factors and gene annotation. However, the results of these analyses remain fragmented, imposing difficulties, either for posterior inspection of results, or for meta-analysis by the incorporation of new related data. Integrating databases and tools into scientific workflows, orchestrating their execution, and managing the resulting data and its respective metadata are challenging tasks. Additionally, a great amount of effort is equally required to run in-silico experiments to structure and compose the information as needed for analysis. Different programs may need to be applied and different files are produced during the experiment cycle. In this context, the availability of a platform supporting experiment execution is paramount. We present GeNNet, an integrated transcriptome analysis platform that unifies scientific workflows with graph databases for selecting relevant genes according to the evaluated biological systems. It includes GeNNet-Wf, a scientific workflow that pre-loads biological data, pre-processes raw microarray data and conducts a series of analyses including normalization, differential expression inference, clusterization and gene set enrichment analysis. A user-friendly web interface, GeNNet-Web, allows for setting parameters, executing, and visualizing the results of GeNNet-Wf executions. To demonstrate the features of GeNNet, we performed case studies with data retrieved from GEO, particularly using a single-factor experiment in different analysis scenarios. As a result, we obtained differentially expressed genes for which biological functions were analyzed. The results are integrated into GeNNet-DB, a database about genes, clusters, experiments and their properties and relationships. The resulting graph database is explored with queries that demonstrate the expressiveness of this data model for reasoning about gene interaction networks. GeNNet is the first platform to integrate the analytical process of transcriptome data with graph databases. It provides a comprehensive set of tools that would otherwise be challenging for non-expert users to install and use. Developers can add new functionality to components of GeNNet. The derived data allows for testing previous hypotheses about an experiment and exploring new ones through the interactive graph database environment. It enables the analysis of different data on humans, rhesus, mice and rat coming from Affymetrix platforms. GeNNet is available as an open source platform at https://github.com/raquele/GeNNet and can be retrieved as software container with the command docker pull quelopes/gennet.

Subjects: Bioinformatics, Computational Biology, Databases, Transcriptome

keywords: GeNNet, Graph database, software container, scientific workflow, transcriptome, microarray, provenance, data-to-knowledge.
\end{abstract}




\section{INTRODUCTION}

The passage of cellular information through the events of transcription and translation postulates the central dogma of molecular biology presented in 1958 by Francis Crick (Crick 1970). Despite the knowledge of the structure of DNA and its main biological functions, it was only in the past few decades, with the advancement of high-throughput technologies, that it became possible to quantify the transcripts produced in large-scale. Since then, important progresses have been noted, for instance, in the identification of prognostic genes and biomarkers, and in the classification and discrimination of subtypes of tumors (Robles and Harris, 2016; Guinney et al., 2015; Alizadeh et al. 2000, Golub et al., 1999; Zhang et al. 2012b). Currently, microarray and RNA-Seq are the main technologies available and widely used (Zhao et al., 2014) in the quantification of gene expression, with advantages and disadvantages in the choice and use of each of them. For instance, on one hand, in RNA-Seq one may both identify new transcripts and observe isoforms (Conesa et al. 2016). On the other hand, the low cost of microarrays, in relation to RNA-Seq, still makes their use very appealing for well-known organisms.

Regardless of the technology employed, the results of transcriptome analysis can be represented as a complex interaction network. In such network, nodes represent transcripts, genes or proteins and the connections between them can be modeled by edges having a weight assigned to them. For example, in gene co-expression networks the links may represent the correlation between the genes limited by a significant value through a cut-off value (Zhang et al. 2012b; Choobdar et al., 2015, Zhang and Horvath, 2005). Strong (positives or negatives) significant correlations among a group of genes may indicate elements that participate in the activation or repression of pathways or biological functions relevant to the studied phenomenon (e.g. immunity, cell differentiation, angiogenesis, etc.). In addition, the same results can be enriched with information from external biological networks such as protein interaction networks (PPI) or even information on identification of key elements in the regulatory process such as the transcription factors (Zhang et al. 2012a; Mathelier et al. 2014). The analysis of the networks may explore topological metrics determining the connectivity between the nodes, of which the most connected can be indicated as targets in molecular modeling, development of biomarkers, etc. Through complex biological networks we can extract topological properties such as 'small-world', 'hierarchically modular' and 'scale-free network' (Barabasi, 2009, Albert, 2005).

Managing such complex network is, however, a challenge. Current approaches employ analysis and visualization software such as Cytoscape (Smoot et al., 2011) and Grephi (Bastian et al., 2009). While such programs make it possible to explore complex relationships between heterogeneous information in biological systems, the results of data analyses often remain fragmented. This imposes difficulties, either for posterior inspection of results, or for meta-analysis by the incorporation of new related data. Furthermore, the heterogeneity of biological data adds to the problem complexity (Maule et al. 2008), as it is hard to find a conceptual data schema that follows a fixed and strict structure, such as in relational databases. Modifying the data schema in these cases can result in conflicts or inconsistencies in a database. In the era of expanding and interconnected information, new data models appeared, such as column-oriented, key-value, multidimensional, and graph databases. These are commonly called NoSQL (Not only $S Q L$ ) databases and often have advantages in terms of scalability (Stonebraker. 2010). Graph-based data models, in particular, are useful for data in which the relationship between attributes is one of the main aspects to be taken into consideration during querying. The graph database is an intuitive way for connecting and visualizing relationships. In graph databases the nodes represent objects and the edges represent the relationships among them. Both, nodes and edges can hold properties, which add information about the objects or the relationships. In recent years, this database model has been used in many bioinformatics applications and are particularly promising for biological datasets (Preusse et al. 2016, Johnson et al. 2014; Balaur et al. 2016; Henkel et al., 2015, Muth et al., 2015, Lysenko et al. 2016). Have and Jensen (Have et al. 2013) observed that for path and neighborhood queries, Neo4j, a graph database, can be orders of magnitude faster than PostgreSQL, a widely used relational database, while allowing for queries to be expressed more intuitively.

Besides the growing need for a more adequate representation for biological data, the accumulation of molecular biology data motivated the development of pipelines, scientific workflows and platforms for analyzing data (Shade and Teal, 2015; Conesa et al., 2016). Many researchers are using these integrative approaches for analyzing metagenomes, proteomes, transcriptomes and other 'omics' data (Joyce and Palsson, 2006). Regardless of the 'omics' technology, there are many steps from the acquisition of raw data to the selection of a subset of representative genes that explain the hypothesis of the scientists. Combining databases and tools into computational analyses, orchestrating their execution, and managing the resulting data and its respective metadata are challenging tasks (Ghosh et al., 2011). Academic journals, for instance, are demanding better reproducibility of computational research, requiring a precise record of parameters, data and processes, also called provenance (Carata et al. 2014), used in these activities to support validation 
by peers (Sandve et al., 2013).

Overcoming many of these challenges can be supported by designing and executing these computational analyses as scientific workflows (Deelman et al. 2009), which consist of compositions of different scientific applications. Their execution is usually chained through data exchange, i.e. data produced by an application is consumed by subsequent applications. Scientific Workflow Management Systems (SWMSs) enable for managing the life cycle of scientific workflows, which is usually given by composition, execution and analysis (Liu et al., 2015). Many SWMSs, such as Galaxy (Giardine et al., 2005), Taverna (Oinn et al., 2004), Tavaxy (Abouelhoda et al., 2012) and Swift (Wilde et al., 2011), natively support reusing previously specified workflows (Goble and De Roure, 2007) and gathering provenance (Gadelha et al. 2012). More recently, scripting languages such as R and Python incorporated features typically available in SWMS. RDataTracker (Lerner and Boose, 2015), for instance, adds provenance tracking to R scripts and noWorkflow (Murta et al. 2015) adds the same functionality to Python. This facilitates the specification and execution of scientific workflows in scripting languages, which is the approach we use in this work. The scientific workflow we propose (GeNNet-Wf) is implemented in R and its activities are comprised of calls to functions of various R libraries, such as limma (Smyth, 2004), GOstats (Falcon and Gentleman, 2007), affy (Gautier et al., 2004) and WGCNA (Langfelder and Horvath, 2008). Integrating scientific workflows with database systems allows for managing and persisting the data manipulated in these workflows in a structured way. This allows for scientists to perform complex data pre-processing analysis and to make the resulting data available for further investigation using queries expressed in a high-level query language. This enables expressing declaratively what data is required without saying how data should be obtained. Moreover, it abstracts away from the user low-level data management details such as accessing files where contents of a database are stored (Garcia-Molina et al., 2009). We argue that integrated web applications, involving scientific workflows and databases, can hide the complexity of underlying scientific software by abstracting away cumbersome aspects, such as managing files and setting command-line parameters, leading to increased productivity for scientists. One important aspect of enabling reproducible computational analyses is keeping track of the computational environment components, i.e., operating system, libraries, software packages and their respective versions (de Paula et al., 2013).

Currently, the vast quantity of functions performed by distinct software lead to a considerable amount of time being employed in installing and configuring them, requiring users to deal with sometimes complicated installation procedures and errors related to software dependencies and versions. Virtualization is a promising technique to tackle these problems (Daniels 2009). In particular, operating system-level virtualization, as provided by 'software containers', allows for running applications and services that are instantiated on isolated environments (containers) on a hosting computer system. Containers provide all the dependencies required for these applications and services to run and can be built in a programmable way to ensure that they will be composed by the same libraries and software packages every time they are instantiated. This considerably facilitates the deployment of software systems since developers can deliver software containers for their applications directly to users or data center administrators. Docker, for instance, is an open-source platform that allows for managing containers (Merkel, 2014; Boettiger, 2015). It has a container repository called Docker Hub ${ }^{1}$ where developers can make software containers for their applications available for download. Many popular software and tools are available on Docker Hub and it is being widely used with around 5 billion software containers downloaded from the repository up to August, $2016^{2}$ In Bioinformatics, there are already tools that are available as Docker software containers and explore features such as reproducibility (Hung et al., 2016, Belmann et al., 2015) or applications areas such as transcriptomics (Zichen et al., 2016). Di Tommaso et al. (2015) showed that containers have negligible impact on the performance of bioinformatics applications. Other examples of software distributed as Docker software containers include: AlgoRun (Hosny et al. 2016), which provides a modular software container with frequently used bioinformatics tools and algorithms that can be accessed through a browser or a Web application programming interface; ReproPhylo (Szitenberg et al. 2015), which implements a phylogenomics workflow with reproducibility features; and GEN3VA (Gundersen et al. 2016), which is a platform for gene-expression analysis available as a web-based system.

Considering other integrative tools for transcriptome data analysis, in the literature there are different integrative approaches for analyzing transcriptomes obtained with from high throughput technologies, such as: Babelomic (Medina et al. 2010), RobiNA (Lohse et al., 2012), Expander (Ulitsky et al., 2010) and RMaNI (Madhamshettiwar et al., 2013). Most of these tools support pre-processing, filtering, clustering, functional analysis, and visualization of results. Furthermore, the tools developed are available for download or as a web interface service. Specific portals for curated bioinformatics tools can be found, for instance, on OmicTools (Henry et al. 2014). However, most of these tools do not

${ }^{1}$ https://hub.docker.com

2 https://blog.docker.com/2016/08/docker-hub-hits-5-billion-pulls/ 
support reproducibility, database management with a flexible and adequate model of representation with persistence, freedom to query the database, and function customization.

In this paper, we present GeNNet, an integrated transcriptome analysis platform that unifies scientific workflows with graph databases for determining genes relevant to evaluated biological systems. It includes GeNNet-Wf, a scientific workflow that accesses pre-loaded back-end data, pre-processes raw microarray data and conducts a series of analyses including normalization, differential expression, gene annotation, clusterization and functional annotation. During these analyses, the results are stored in different formats, e.g., figures, tables and $\mathrm{R}$ workspace images. Furthermore, experiment results are stored in GeNNet-DB, which is a graph database that can be persisted. The graph database represents networks that can be explored either graphically or using a flexible query language. Finally, GeNNetWeb offers an easy-to-use web interface tool developed in Shiny for automated analysis of gene expression. The implementation follows best practices for scientific software development (Wilson et al., 2014). For instance, our approach uses both software containers and provenance tracking to facilitate reproducibility. This allows for reproducing, without user intervention, the computational environment (e.g. operating system, applications, libraries) and recording the applications, data sets and parameters used in the analyses, i.e. tracking data provenance. A graph data model is used to adequately represent gene expression networks and its persistence. Also, a high-level declarative language can be used to freely query the data, existing functions can be modified and new functions added to the analytical workflow. As far as we know, GeNNet is the first platform for transcriptome data analysis that tightly couples a scientific workflow with a persistent biological (graph) database while better supporting reproducibility.

To emphasize and demonstrate the usefulness of GeNNet, we will reanalyze data from hepatocellular carcinome (HCC) in tumor versus adjacent non-tumorous liver cells under different scenarios of use and analysis from GEO repository (Gene Express Omnibus). The first one (I), consists of executing the experiment using the user-friendly interface of GeNNet in which users choose the parameters and execute the experiment without needing to modify the lower-level scripts that compose GeNNet. The second one (II) is comprised of integrating data from different experiments that have the same hypothesis to be tested and evaluated using the RStudio IDE. The third and last one (III) uses the results of scenarios (I) and (II) to perform queries in the graph database. In (III), we highlight the use of the database persisted during the execution of GeNNet, as well as the integration of new information into it.

\section{MATERIALS AND METHODS}

\section{Implementation}

GeNNet innovates in its use of a graph-structured conceptual data model coupled with scientific workflow, software containers for portability and reproducibility, and a productive and user-friendly web-based front-end (Figure 1). In the following subsections, we describe these components and functionalities in detail: scientific workflow (GeNNetWf), graph database (GeNNet-DB), web application (GeNNet-Web), software container, computational experiment reproducibility and experimental data.

\section{GeNNet-Wf workflow}

GeNNet-Wf is the composition of two sub-workflows: 'Background workflow' and 'Analysis workflow' (see in Figure 2). The data obtained by the former persists into the graph-database. The 'Analysis workflow' processes the raw dataset enriching the background data.

\section{Background workflow}

The GeNNet 'Background workflow' generates a database for a set of specified organisms pre-loaded into the system (Figure 2, top). It includes genes annotated/described and their relationships, along with other associated elements, that contribute to posterior transcriptome analysis. In this version of the platform, the background data is comprised of two main sources: (i) gene information about human, rhesus, mice and rat, obtained from NCBI annotations (Schuler et al. 1996); and, (ii) Protein-Protein Interaction (PPI) network, retrieved from STRING-DB (Franceschini et al., 2013) (version 10). All genes imported from NCBI become nodes in the graph database and some of the main information associated to them (such as symbol, entrezId, description, etc.) are modeled as node properties. The information derived from STRING-DB PPI become edges ('neighborhood', 'gene fusion', 'co-occurrence', 'co-expression', 'experiments', 'databases', 'text-mining' and 'combined score'). This layer of data is added to the graph database during the construction of the GeNNet container (Software container subsection). More detail about the representation and implementation can be found in section GeNNet-DB graph database. 


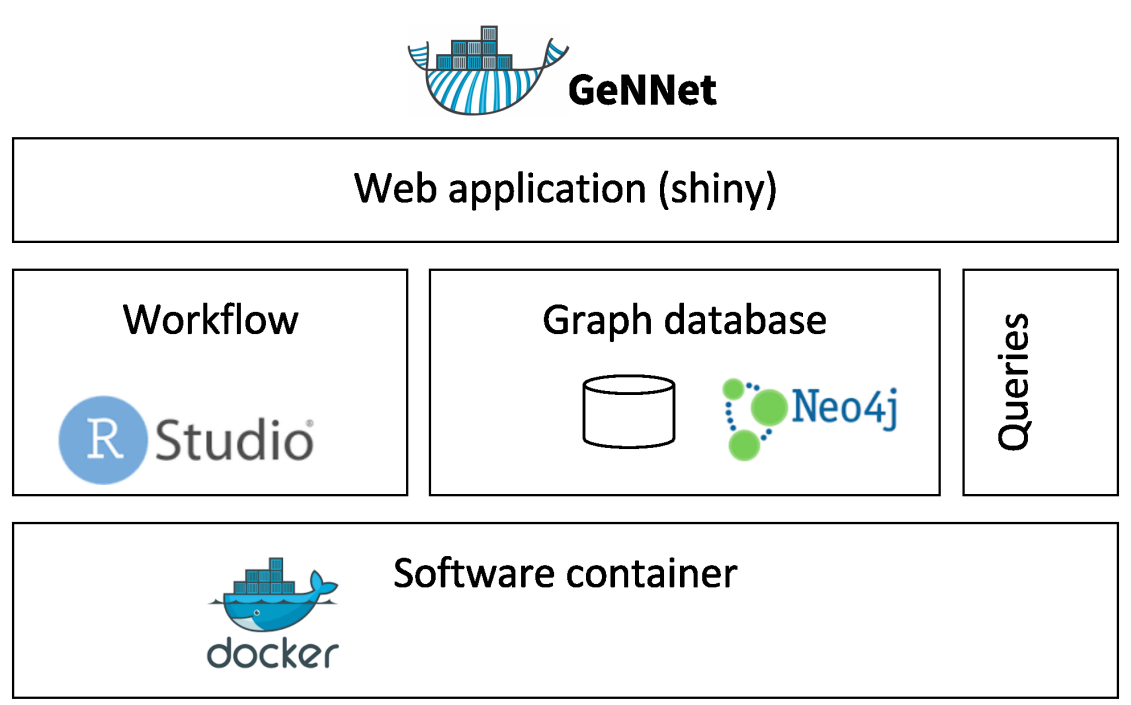

Figure 1. GeNNet framework with its components: Scientific Workflow (GeNNet-Wf), Graph database (GeNNet-DB) and user-friendly interface (GeNNet-Web).

\section{Analysis workflow}

The 'Analysis workflow' stage is comprised of the execution of a series of tools and libraries to analyze the transcriptome data uploaded by the user in conjunction with the data generated by the 'Background workflow' (Figure 2, bottom). This module was written in R using several packages mainly from the Bioconductor (Dudoit et al., 2003) and CRAN repositories. The steps are detailed next.

Normalization. This step consists in normalizing the raw data from an informed Affymetrix platform using either RMA (Irizarry et al. 2003) or MAS5 methods, both available in the affy (Gautier et al., 2004) package. During this step, some quality indicator plots are generated (as boxplot of probe level, Spearman correlation and density estimates) as well as a normalized matrix (log-normalized expression values).

e-set. In this step, data about the experimental design should be added along with log-normalized expression values. This generates an ExpressionSet (eSet) object, a data structure object of the S4 class used as base in many packages developed in Bioconductor transcriptome analysis (Falcon et al., 2007). This format gives flexibility and access to existing functionality. The input file must be structured using mainly two columns: a column named SETS for the experimental design, and a column named SAMPLE_NAME for the names of the files containing raw sample expression matrix data.

Filtering/Differential expression inference. Differential expression (DE) inference analysis allows for the recognition of groups of genes modulated (up- or down-regulated) in a biological system when compared against one or more experimental conditions. In many situations this is a core step of the analysis and there is a great diversity of experimental designs (such as control versus treatment, consecutive time points, etc) allowing the inference. In our platform, we use the limma package to select the DE genes (Smyth. 2004) on single-factor experimental designs based on a gene-based hypothesis testing statistic followed by a correction of multiple testing given by the False Discovery Rate (FDR) (Kendall and Bradford Hill 1953). Furthermore, a subset of DE genes can be selected based on a upand down-regulation, expressed as an absolute logarithmic (base 2) fold-change (logFC) threshold. The latter can be set-up by the user, as described in Scenario I - Experiment user-friendly interface. Results of this step are displayed as Volcano plots and matrices containing the DE genes.

Annotation. The annotation step consists of annotating the probes for the corresponding genes according to the Affymetrix platform used in the experiment.

Clusterization. This step consists in analyzing which aggregated genes have a similar pattern (or level) of expression. We incorporated clusterization analysis including hierarchical methods, $k$-medoids from the package PAM (Partitioning 


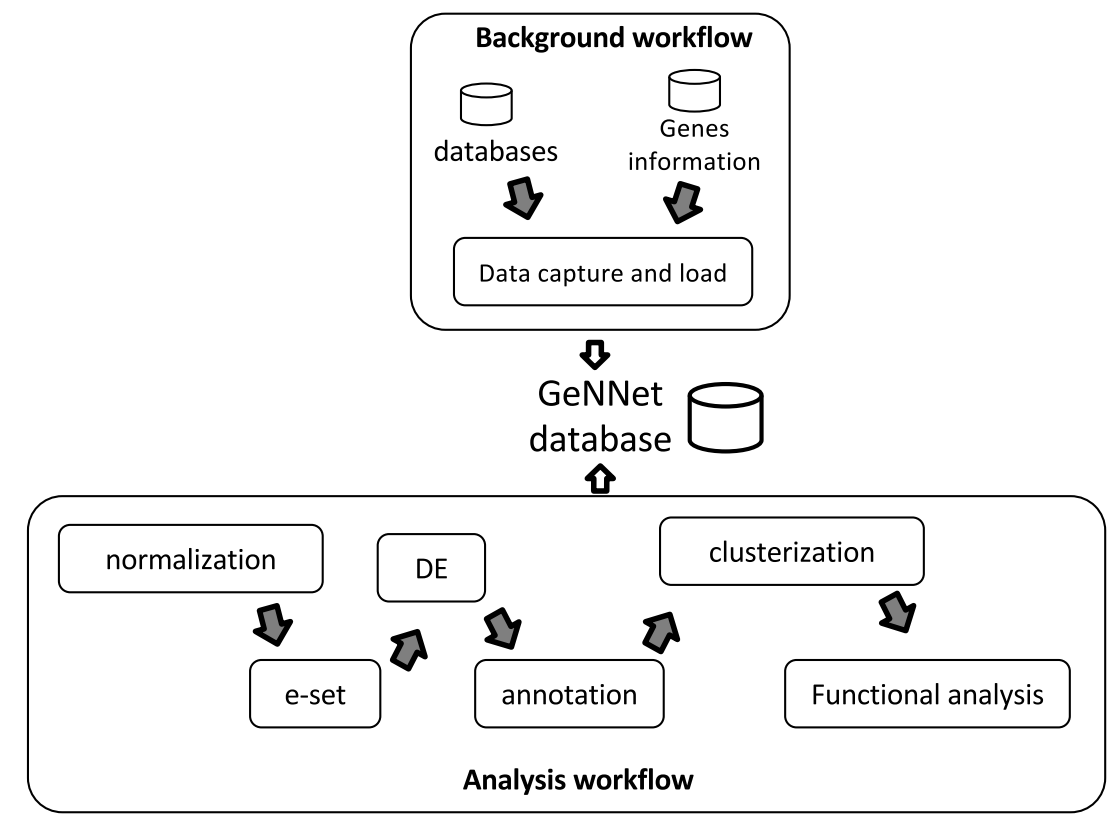

Figure 2. Workflow scheme represented by two stages, 'Background workflow' in the top and 'Analysis workflow' in the bottom. Results of both stages are loaded to the GeNNet database. The 'Analysis workflow' stage is shown with its different steps of the analysis. e-set is the Expression Set and DE is the Differential Expression.

Around Medoids) (Reynolds et al. 2006) and WGCNA (Weighted Gene Coexpression Network Analysis) (Langfelder and Horvath, 2008).

Functional Analysis. Genes grouped by similar patterns enable the identification of over-represented (enriched) biological processes (BP). In our approach, we conducted enrichment analyses applying hypergeometric tests (with p-value $<0.001$ ) as implemented in the GOStats package (Falcon and Gentleman, 2007). Ontology information for the gene is extracted from the Gene Ontology Consortium database (Ashburner et al. 2000). The universe is defined as the set of all genes represented in a specific Affymetrix platform, or, in case of multiple platforms in a single experiment design, the universe is defined as the common and unique genes in among all Affymetrix platforms. The subset is defined either by the set of diferentially expressed (DE) genes between a test and a control condition (control versus treatment design), or by the union of the DE genes selected among the pairwise comparisons among groups in all other single-factor experimental designs. Although functional analyses can lead to biased results, as presented in Timmons et al. (2015), we have added more restrictive cut-off with the purpose of reducing the detection bias of our platform.

\section{Execution}

GeNNet is designed to automatically execute the workflow through the web application interface (accessed via: http: //localhost:3838/gennet, when the software container is running). However, users that intend to implement new functions or even execute the workflow partially, can use the RStudio server interface in GeNNet (accessed via: http://localhost: 8787 after starting the software container). More details are available in Supplemental Information.

\section{GeNNet-DB graph database}

Although a NoSQL database has no fixed schema, we defined an initial graph model to help and guide the GeNNetDB (Figure 3). GeNNet database (GeNNet-DB) structure is defined on the Neo4j database management system, a free, friendly-to-use and with broad community support graph database, with its nodes, edges and relationships. Vertices and edges were grouped into classes, according to the nature of the objects. We defined the labels as GENE, BP (Biological Process), CLUSTER, EXPERIMENT, ORGANISM, and a series of edges as illustrated in Figure 3 . In the GeNNet platform there is an initial database defined by interactions between genes as described in Background preparation section. During the execution of GeNNet-Wf, using Shiny or RStudio, new nodes and connections are formed and 
added to the database. The resulting information is stored in the graph database using the RNeo4j packag $\oint^{3}$ It can also be accessed directly through the Neo4j interface (accessed via: http://localhost:7474). It is possible to query and access the database in this interface using the Cypher language, a declarative query language for Neo4j, or Gremlin, a general-purpose query language for graph databases. These query languages allow for manipulating data by updating or deleting nodes, edges and properties in the graph. Querying also allows for exploring new hypotheses and to integrate new information from different resources that are related to the targeted experiment. GeNNet-DB is persistent and the resulting database is exported to a mounted directory. Its contents can be loaded to a similar Neo4j installation. For further details one can read the Neo4j manual.

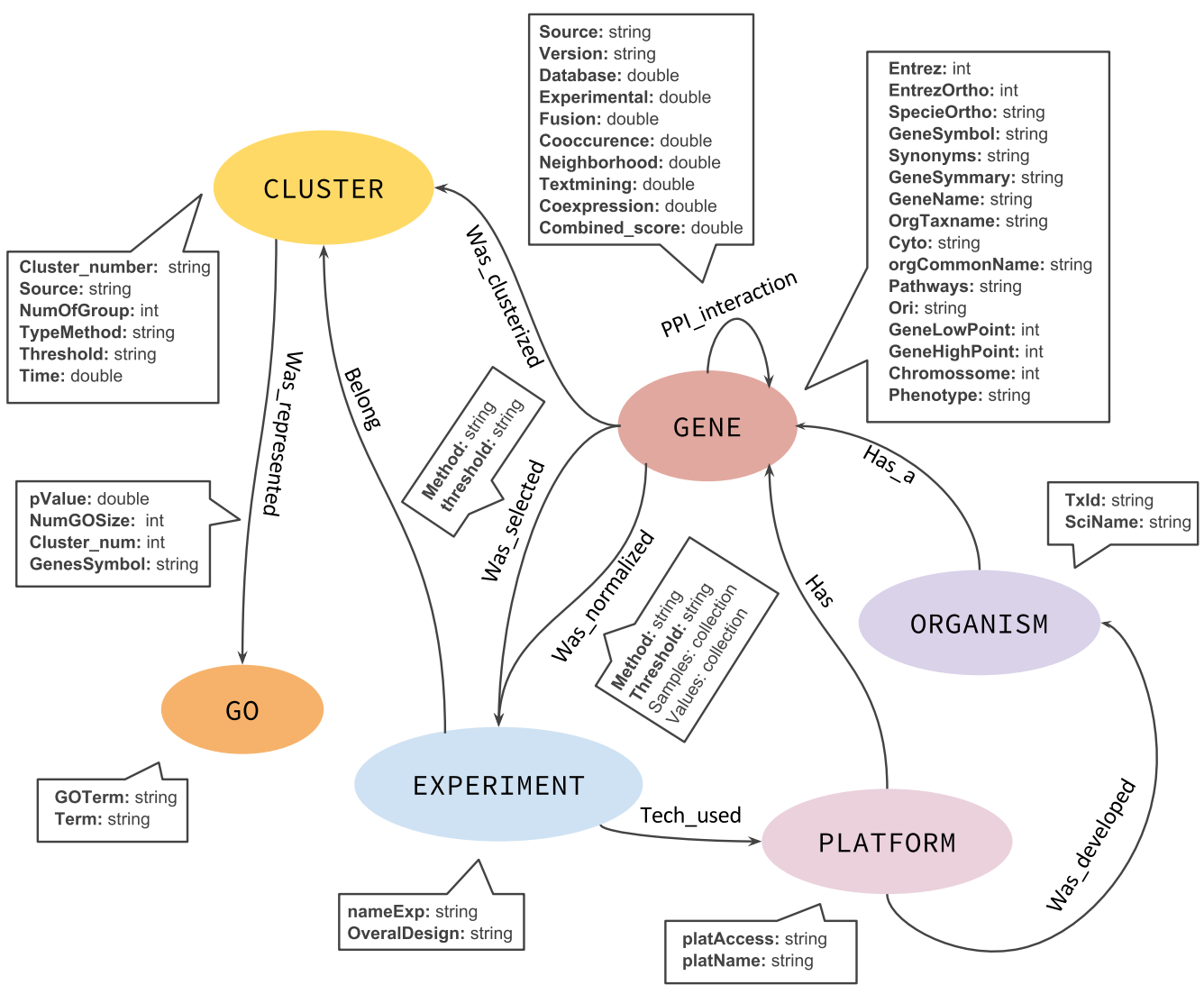

Figure 3. The graph model representing the nodes (in oval), relationships (arrows). The descriptive boxes showing the mainly properties in nodes and edges.

\section{GeNNet-Web application}

GeNNet-Web provides a user-friendly way to execute GeNNet-Wf. We developed an easy-to-use layout for providing the parameters and automatically executing all steps of the workflow experiment. The application was implemented using the Shiny library for R. This library allows for turning an R script that implements some analysis into a web application in a convenient manner. Shiny has a library of user interface elements can be used to entering input data and parameters, and for displaying the output of an R script. The parameters comprise the input of the web application, which include: descriptors for experiment name and overall design; type of normalization; differential expression settings; experiment platform and organism; and clusterization method. After executing GeNNet-Wf, GeNNet-Web allows for easy retrieval and visualization of its outputs, which are given by a heatmap, graph database

${ }^{3}$ Available at: https://cran.r-project.org/web/packages/RNeo4j 
metrics (e.g., number of nodes, number of edges and relationships between nodes), and the list of differentially expressed genes selected. In addition to the outputs generated in the web application, the underlying workflow generates the output files described in subsection GeNNet-Wf workflow.

\section{Software container}

A Docker software container was built containing GeNNet an all its requires libraries and dependencies. This enables users to download a single software container that includes all the components of GeNNet and instantiate this environment independently any of the host that runs an operating system supported by Docker. The software container was successfully tested on CentOS Linux 7, Ubuntu Linux 14.04, MacOS X 10.11.6 and Windows 10 hosts. The software container for GeNNet, specified in a script named 'Dockerfile', was built according to the following steps: (i) The operating system environment is based on Debian GNU/Linux 8 with software packages required by GeNNet, such as R (v. 3.3.1), installed from the official Debian repositories; (ii) The R software and the packages required by GeNNet, installed from the CRAN repository; (iii) RStudio (v. 1.0.44) server and the Neo4j (Community Edition v.3.0.6) graph database, installed from their respective official repositories; (iv) Supporting data sets, such as PPI, loaded to the graph database; (v) GeNNet-Wf, implemented in R, installed in RStudio; (vi) Shiny, a web application server for R, installed from its official repository. GeNNet-Web, which calls GeNNet-Wf, is loaded to Shiny.

\section{Computational experiment reproducibility}

Reproducibility is accounted in GeNNet in two aspects. Firstly, the platform provides a provenance trace record generated by the RDataTracker package (Lerner and Boose. 2015) for R. The trace contains the activities executed by the workflow and the data sets consumed and produced by them. This trace is exported to a persistent directory. Secondly, the adoption of software containers allows for using the same environment (operating system environment, libraries, and packages) every time GeNNet is instantiated and used. Both the provenance trace and the preservation of the execution environment with software containers significantly help the computational experiment reproducibility since users can retrieve from the former the parameters and data sets used in analyses and, from the latter, re-execute them in the same environment, as provided by the GeNNet software container.

\section{Experimental data - Use case scenarios}

To illustrate the flexibility of GeNNet, we will conduct an experiment of re-analysis of HCC, considered the most common type of liver cancer. The HCC is highly complex and the main risk factors are associated with prolonged abusive alcohol consumption and persistent infection of HBV (Hepatitis B Virus) and HCV (Hepatitis C Virus) (Siegel et al. 2017). We performed the re-analysis of microarray experiments deposited in the GEO repository and, to facilitate the understanding of GeNNet, we separated this case study in three different scenarios. The first one is to analyze the data using the friendly web interface for GeNNet developed in Shiny (described in subsection GeNNet-Web application). The second scenario is to integrate an additional independent experiment to the data using the RStudio interface to create and/or modify their own functions. The last scenario is to perform queries in the graph database generated during the execution and analysis of the experiment, highlighting the range of possibilities of the system we developed.

\section{RESULTS AND DISCUSSION}

\section{Scenario I - Experiment user-friendly interface}

As an example of a specific and more detailed case study, we re-analyzed a gene expression experiment from HCC obtained from the transcriptome repository GEO (Barrett et al. 2013) with accession number GSE62232 (Schulze et al. 2015). The study used the Affymetrix Human Genome U133 Plus (GPL570) and contains 91 samples, of which 81 samples are from HCC tumors and 10 from adjacent non-tumorous liver tissues.

Data was normalized using the MAS5 method and the differentially expressed gene selection criteria were FDR $<$ 0.05 and absolute $\log 2$ (Fold-Change) $>1$. The initial threshold values chosen are the most used and recommended in the literature but the threshold values can be adjusted. The genes were clustered using the Pearson correlation method as measure of dissimilarity. Next, the clusters were associated to biological functions through the hypergeometric test (with p-value $<0.001$ as threshold). All parameters were configured using the friendly interface built in Shiny as shown 


\section{GeNNet Platform}

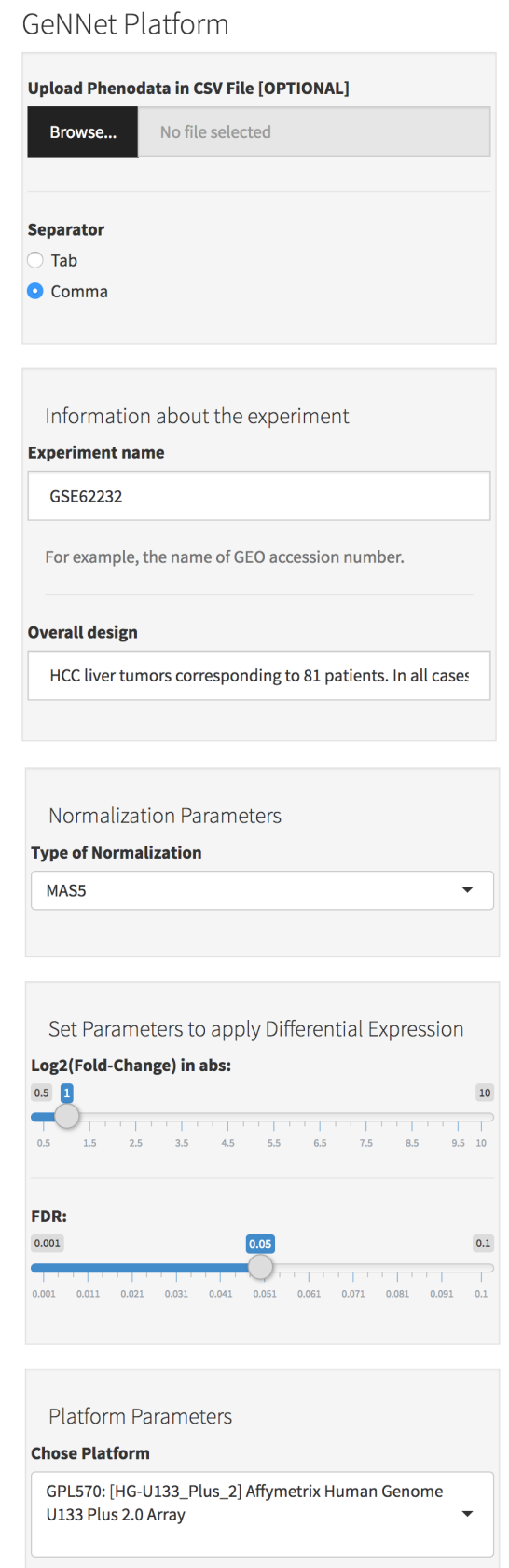

in Figure 4 and accessed via http: / / localhost:3838/gennet As a result, 3356 differentially expressed genes were obtained and 661 ontological terms were represented (p-value $<0.001$ ). A major part of the information resulting from the analytical process was incorporated to GeNNet-DB and besides the database, the results were exported to different formats such as figures (heatmaps, boxplots, etc.), tables and provenance (Figure 5).

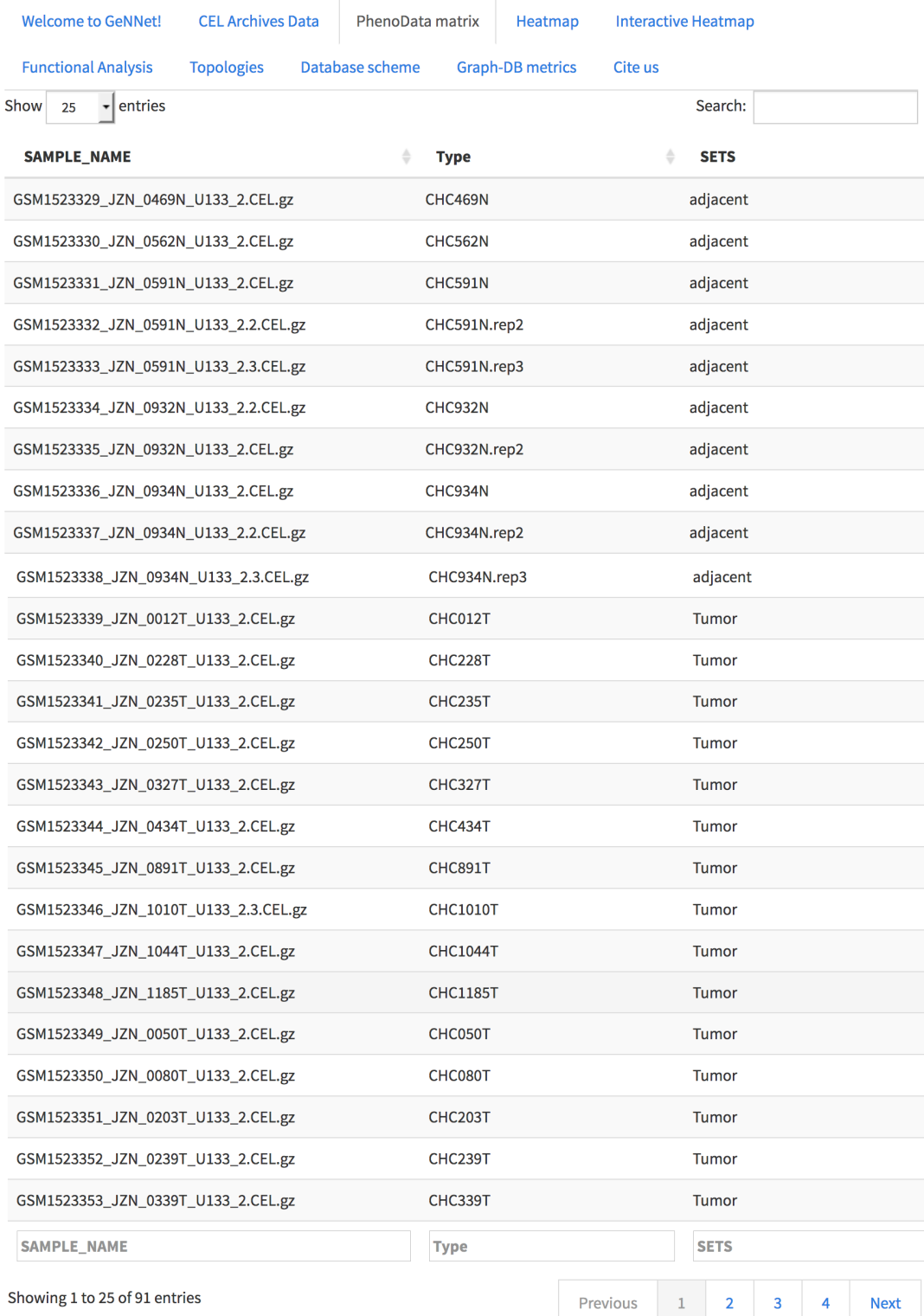

Figure 4. User-friendly interface in GeNNet. Left-hand side showing parameters settings and right-hand side showing some tabs highlight PhenoData matrix uploaded.

\section{Scenario II - RStudio environment in meta-analysis}

In this scenario we explored the flexibility introduced by the integration of RStudio in our platform. Its availability enables more experienced users to extend existing functionality with new analyses over available data. In this 


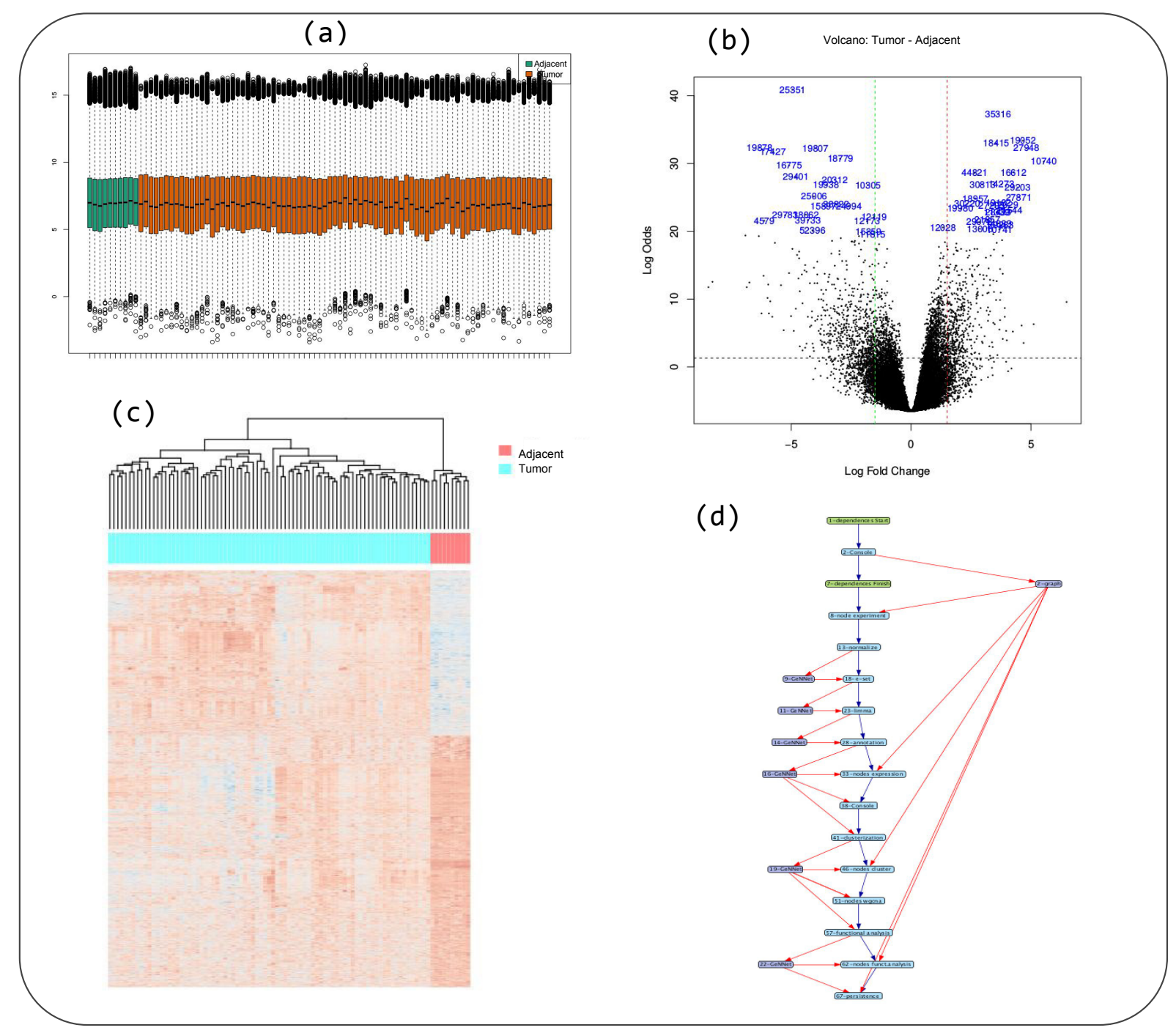

Figure 5. Some captions of figures generated during workflow execution: (a) boxplot of quality indicator, (b) volcano plot, (c) heatmap of genes differentially expressed in tumor versus adjacent, and (d) the provenance trace of a GeNNet-Wf execution is represented as a data derivation graph (DDG).

scenario, we explore one example of such flexibility with a meta-analysis approach in which we combine results from different experiments. Meta-analysis experiments combine microarray data from independent yet similarly designed studies allowing one to overcome their variations, and ultimately increasing the power and reproducibility of the transcriptome (Ewald et al. 2015) analysis. We added a study with experimental design performed on the data described in the previous section. We used HCC data containing 18 tumor samples versus 18 adjacent non-tumorous liver tissues from Wang et al. (2014). The experiment was carried out with the Affymetrix Human Genome U133 platform and deposited in GEO under accession number GSE60502. The Figure 6 shows the access via RStudio (accessed via: http: \localhost:8787).

This scenario of use requires more advanced users in the $\mathrm{R}$ language. We exemplify the addition of an experiment to enhance the flexibility of our platform by making the analysis more robust and integrative between complex experiments as in cancer studies. However, the user can modify or even add a function by generating new analyses from GeNNet. 


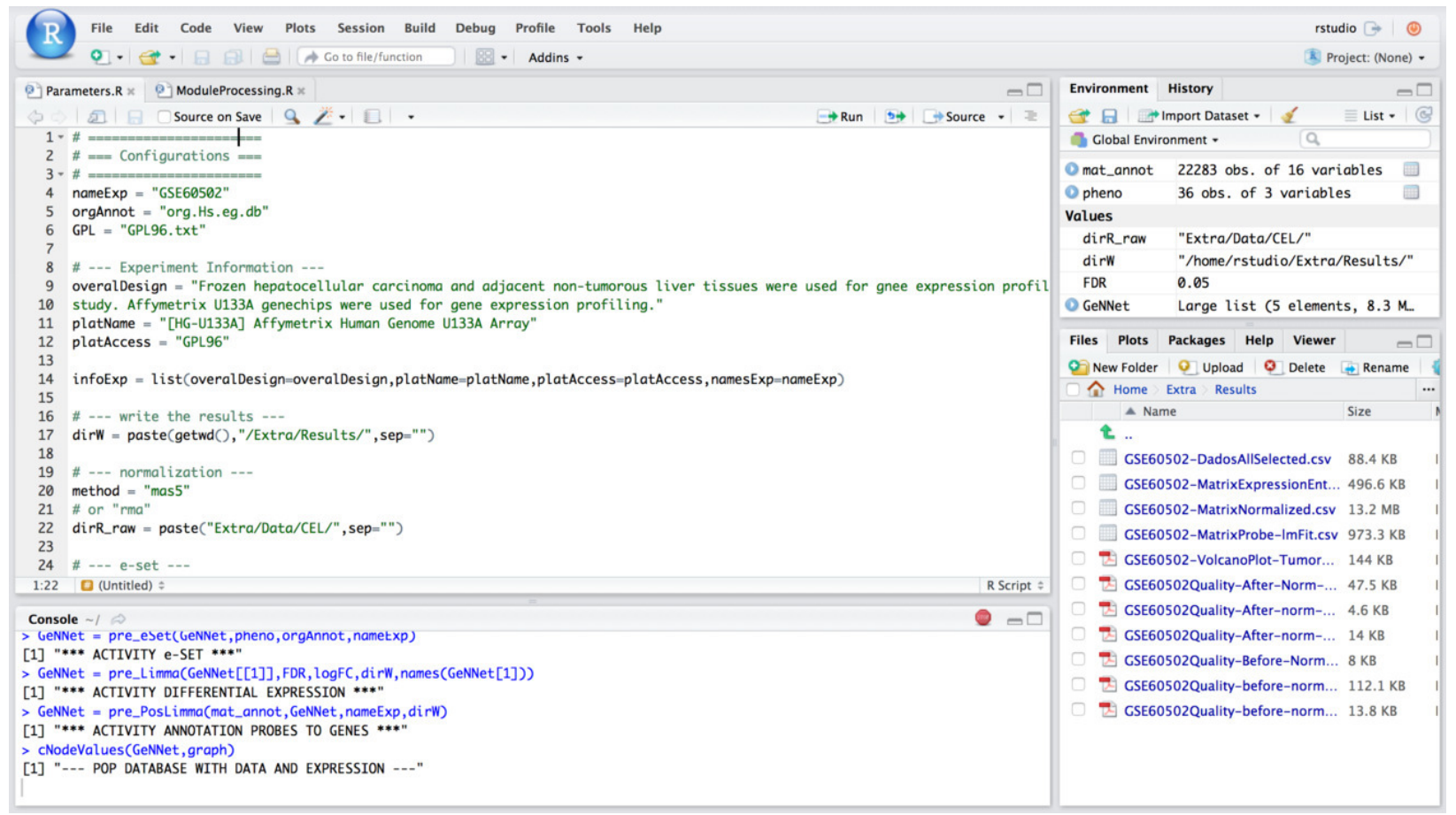

Figure 6. Example of access mode to RStudio where it is possible to modify the predefined functions.

\section{Scenario III - Querying and adding relationships}

Biological information is typically highly connected, semi-structured and unpredictable. The results obtained from the GeNNet analysis are stored in a graph database during the execution of the workflow. The database can be accessed via http://localhost:7474 using the Cypher declarative query language with direct access to the database, we formulated some demonstration queries using as example the dataset analyzed above. The database generated during GeNNet-Wf execution facilitates data representation as interaction networks, in an approach that allows for exploring a great variety of relationships among its composing entities, besides making new insights for subnetwork exploration possible. Depending on the type of these interactions, different kinds of networks and topologies can be defined and analyzed. Through the data representation used in GeNNet-DB, traversal queries are possible. We illustrate typical examples in which the user just needs to query GeNNet-DB to solve them.

Query 1: What are the existing relationships among nodes in the database?

This is a simple query that returns all existing relationships among different node labels and types. The result of the query was represented as a graph in Figure 7 retrieved the graph model as exemplified in Figure 3.

MATCH (a) $-[\mathrm{r}]-(\mathrm{b})$

WHERE labels (a) $<$ [] AND labels (b) $<$ []

RETURN DISTINCT head (labels (a)) AS This,

type (r) as Relation,

head (labels (b)) as To

Query 2: Which nodes of type GENE were DE and present the highest number of connections associated to the protein interaction networks (PPI) according to a combined score threshold of $>0.80$ ? Among these selected nodes, what are the clusters and associated biological processes?

Some common and important topological metrics in biological networks include: degree, distance, centrality, clustering coefficient. In this work, we use the degree metric $k_{i}$ of a node $n_{i}$, defined as the number of edges that are adjacent $\left(a_{i j}\right)$ to this node, which is given by:

$$
k_{i}=\sum_{j \in V} a_{i j} .
$$

We use the Cypher query language to find the most connected DE genes in the network that establish known 

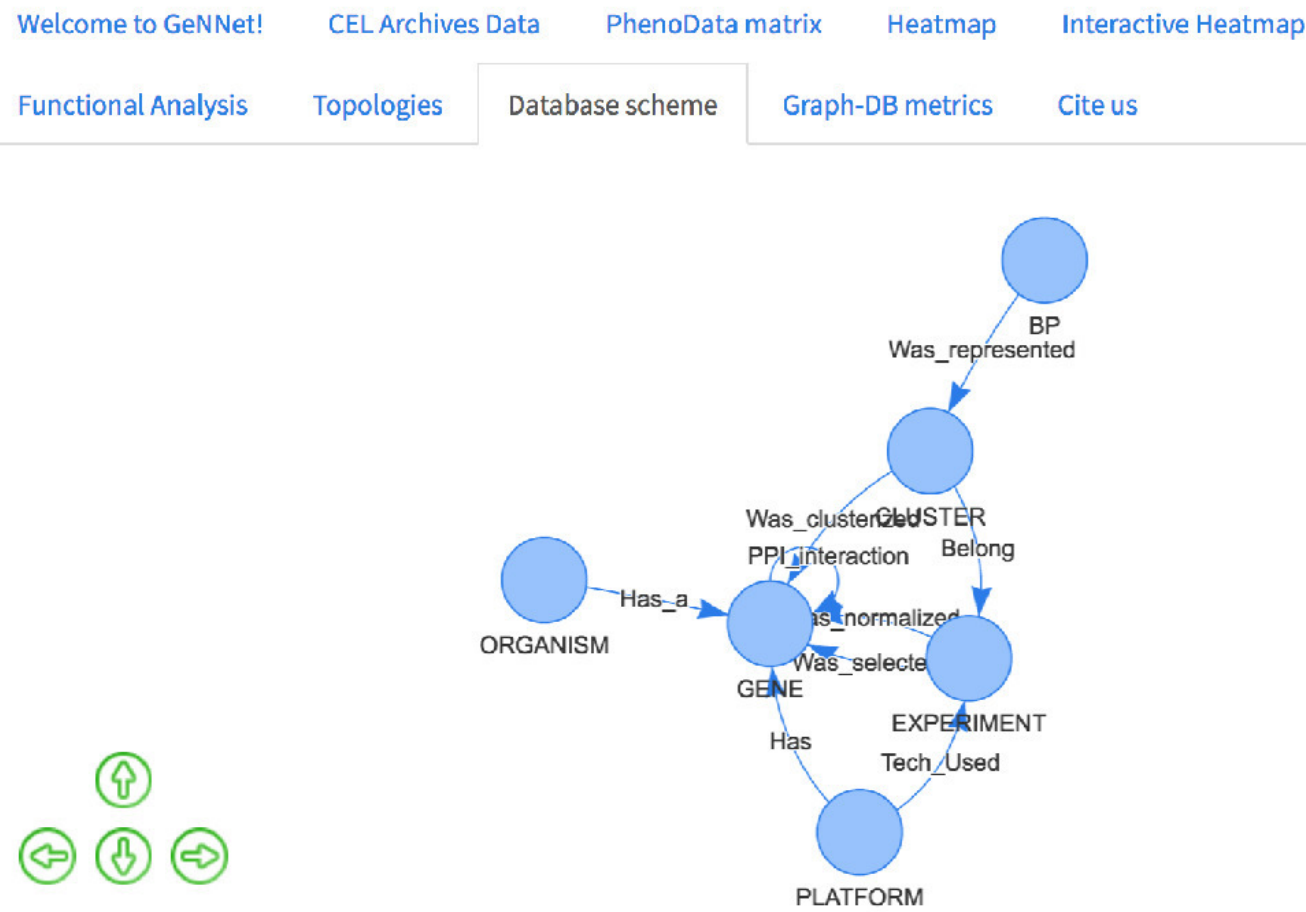

Figure 7. Database schema with all the existing nodes and relationships.

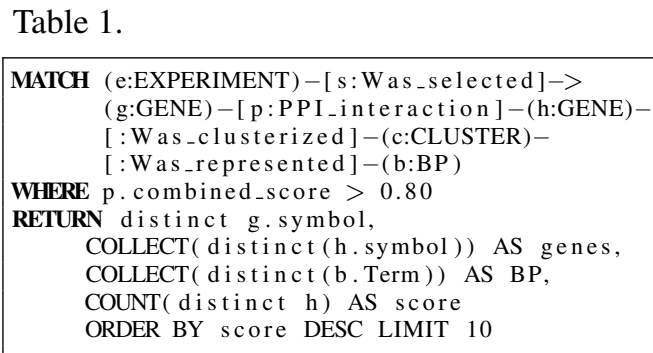
Table 1.

connections to the PPI network, having a high attribute value for the combined interaction score (provided by PPI association of protection interaction database STRING-DB). For these genes we computed the co-expression cluster and, subsequently, the biological processes attributed to these clusters. One can observe that the query is expressed in a concise manner for answering a relatively complex topological question. The resulting DE genes are displayed in

One of the main advantages of using the data model adopted in GeNNet is the availability of data and information that can be easily done without changing the data model. New nodes may add information such as metadata of samples (e.g. information on a patient's eating habits) or new edges may add new relationships (e.g. genes co-expressed in different methods used) or even both (e.g. addition of a database on microRNA interactions connected to existing genes in the database). In the example below, we add a HUB-like node from the result obtained in query 2 . Through the CREATE clause, after obtaining the selected genes, a new node and edges were created (Figure 8). This queries demonstrates the flexibility of the database in adding new information that can be generated through existing data in GeNNet-DB. 
Table 1. Result showing the top 10 gene DE by PPI in experiment GSE62232. These genes are known as hubs and may be associated with important pathways in the experimental context analyzed.

\begin{tabular}{|c|c|c|}
\hline $\begin{array}{l}\text { Genes } \\
\text { selected }\end{array}$ & $\operatorname{cgn}^{a}$ & BP associated \\
\hline CDK1 & 89 & \multirow{10}{*}{$\begin{array}{l}\text { regulation of tau-protein kinase activity; } \\
\text { L-cysteine metabolic process; negative regulation } \\
\text { of natural killer cell differentiation; response to } \\
\text { lipopolysaccharide; positive regulation of } \\
\text { angiogenesis; regulation of lipid metabolic process }\end{array}$} \\
\hline SRC & 92 & \\
\hline PLK1 & 83 & \\
\hline JUN & 73 & \\
\hline BIRCS & 68 & \\
\hline AURKB & 68 & \\
\hline FOS & 66 & \\
\hline PCNA & 61 & \\
\hline ADCYS & 60 & \\
\hline POMC & 60 & \\
\hline
\end{tabular}

${ }^{a}$ number of connected genes

Query 3: New node and edges inserted from the result of the previous query.

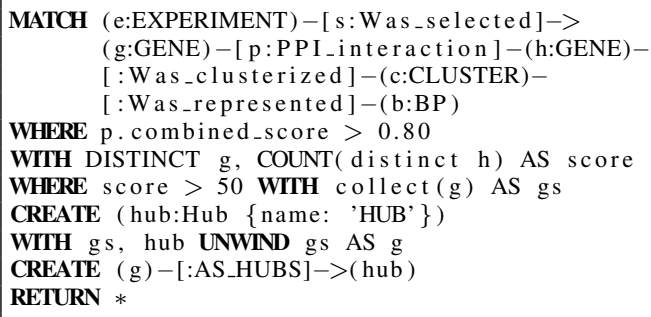

\section{*(20) GENE(19) Hub(1) \\ *(136) AS_HUBS(19) PPI_interaction(117)}

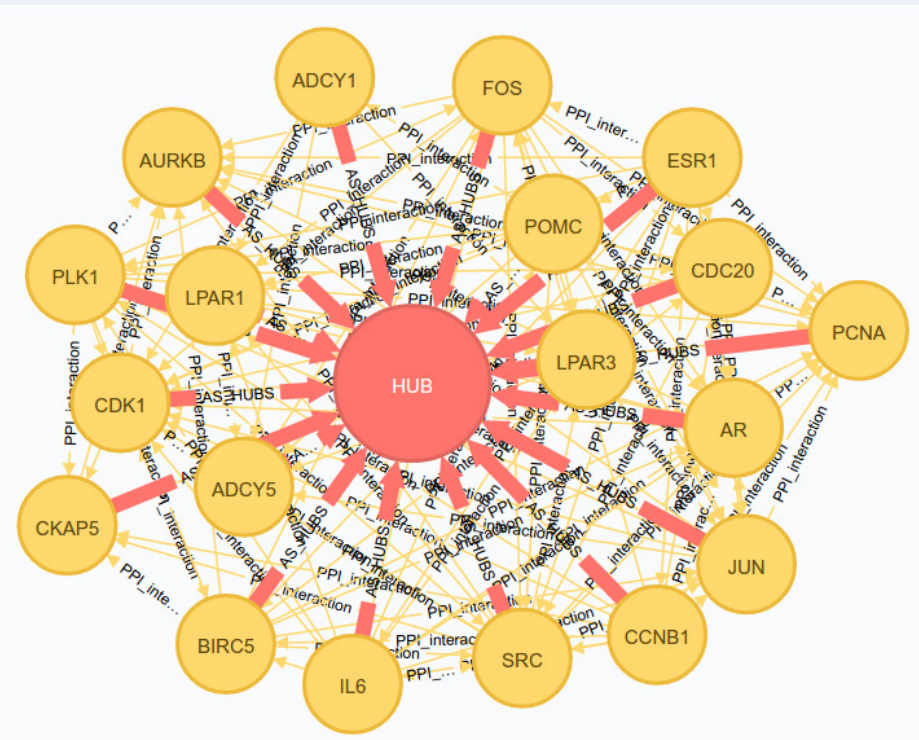

Figure 8. New nodes and edges added to the graph database. The genes that were highly connected according to query 3 were directed to a node of type HUB. 


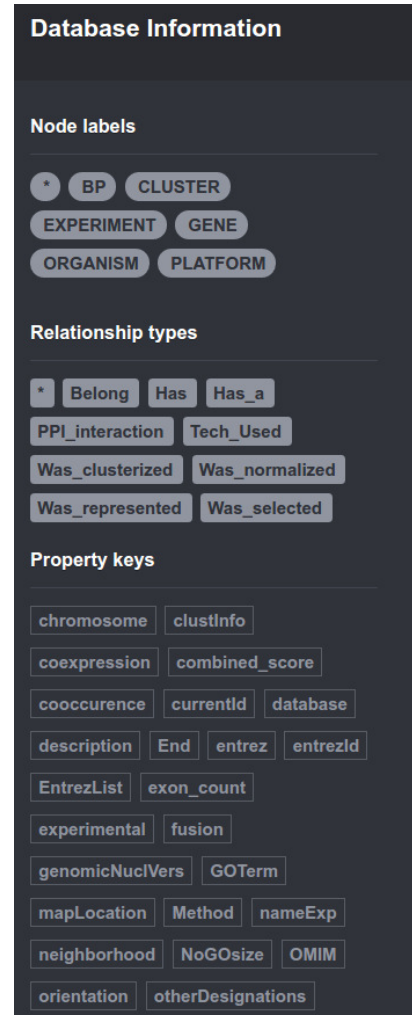
instance, gene 'CDK1'?

WHERE g . symbol='CDK1'

ORDER BY score DESC

Query 4: Given different experiments, which genes are co-expressed with a differentially expressed gene, for

Through this query we can know which genes are co-expressed with CDK1 ranked in descending order on the number of experiments analyzed and deposited in the database. As a result from this query, we obtain that 326 genes appear co-expressed with gene CDK1 in both experiments analyzed in scenarios I and II (Figure 9).

MATCH (g:GENE) $-[\mathrm{r}: \mathrm{W}$ a s_clusterized $]->(\mathrm{c}:$ CLUSTER $)<-[\mathrm{r} 2:$ W as _clusterized $]-(\mathrm{h}:$ GENE $)$

RETURN h.symbol,count (distinct c) AS score

collect (distinct c.clustinfo) as cluster

Figure 9. Result of query 4 in the Neo4j access interface displaying the genes co-expressed with CDK1 in the different experiments deposited in GeNNet-DB.

MATCH (g:GENE) - [r:Was_clusterized] -> (c:CLUSTER)<-[r2:Was_clusterized $]-(\mathrm{h}:$ GENE) WHERE $g$. symbol $=$ ' CDK1

RETURN h.symbol, count (distinct c) AS score, collect (distinct c.clustinfo) as cluster ORDER BY score DESC

$\$$ MATCH $(g: G E N E)-[r$ :Was_clusterized $]->(c:$ CLUSTER $)<-[r 2$ :Was_clusterized $]-(h: G E N E)$ WHERE g.symbol='CDK1' RETURN $\mathrm{r}$ 曲 h.symbol score cluster

Rows IRGQ

CGREF1

PRIM1
LOC81691

SPINK1

SMARCA4 2

BUB1

RAD51AP1 2

OIP5

GPSM2 2

LAMC1 2

PRUNE

LPHN1

CENPM 2

RNF144A

MCM10

[C_2_GSE60502_average-Pearson, C_2_GSE62232_average-Pearson] [C_2_GSE60502_average-Pearson, C_2_GSE62232_average-Pearson] [C_2_GSE60502_average-Pearson, C_2_GSE62232_average-Pearson] [C_2_GSE60502_average-Pearson, C_2_GSE62232_average-Pearson] [C_2_GSE60502_average-Pearson, C_2_GSE62232_average-Pearson] [C_2_GSE60502_average-Pearson, C_2_GSE62232_average-Pearson] [C_2_GSE60502_average-Pearson, C_2_GSE62232_average-Pearson] [C_2_GSE60502_average-Pearson, C_2_GSE62232_average-Pearson] [C_2_GSE60502_average-Pearson, C_2_GSE62232_average-Pearson] [C_2_GSE60502_average-Pearson, C_2_GSE62232_average-Pearson] [C_2_GSE60502_average-Pearson, C_2_GSE62232_average-Pearson] [C_2_GSE60502_average-Pearson, C_2_GSE62232_average-Pearson] [C_2_GSE60502_average-Pearson, C_2_GSE62232_average-Pearson] [C_2_GSE60502_average-Pearson, C_2_GSE62232_average-Pearson] [C_2_GSE60502_average-Pearson, C_2_GSE62232_average-Pearson] [C_2_GSE60502_average-Pearson, C_2_GSE62232_average-Pearson]

Returned 1589 rows in 702 ms, displaying first 1000 rows.

\section{CONCLUSION, UPDATES AND FUTURE WORK}

The platform presented in this work is the first one to integrate the analytical process of transcriptome data (currently only available for microarray essays) with graph databases. The results allow for testing previous hypothesis about the experiment as well as exploring new ones through the interactive graph database environment. It enables the analysis of different data coming from Affymetrix platforms on humans, rhesus, mice and rat. GeNNet will be periodically updated and we intend to extend the modules to include analyses of RNA-Seq and miRNA. We will incorporate additional experimental designs for DE and improve the execution time of the analyses. Moreover, we intend to add other model organisms to the background data, such as Arabidopsis thaliana and Drosophila melanogaster.

GeNNet-Web offers an interface that accommodates both experienced and inexperienced users. For the latter, the interface provides various filtering and parameter setup opportunities, in addition to some pre-defined queries. For more advanced users a plain query interface is provided so that more tailored analysis can be expressed. Due to the free access to GeNNet, we rely on the feedback of the community for improving the tool. The distribution of the platform in a software container allows not only for executing it on a local machine but also for easily deploying it on a server and making it available on the Web. 


\section{ADDITIONAL INFORMATION AND DECLARATIONS}

\section{Funding}

This work has been supported by CAPES (Coordenação de Aperfeiçoamento de Pessoal de Nível Superior) and CNPq (Conselho Nacional de Desenvolvimento Científico e Tecnológico) funding.

\section{Competing Interests}

The authors declare that they have no competing interests.

\section{Author Contributions}

RLC conceived idea, the software architecture, designed the graph query language and writing the manuscript. The LG worked in the software container implementation, the GeNNet architecture proposal, and on writing the manuscript. MRA and FP writing the manuscript and provided critical comments improved upon the manuscript and application. All authors read and approved the final version of then manuscript.

\section{Data Availability}

GeNNet's source code is available at https://github.com/raquele/GeNNet. A software container that allows for easily executing GeNNet can be retrieved from/https://hub.docker.com/r/quelopes/gennet/.

\section{Supplemental Information}

GeNNet manual is available in supplementary material.

\section{REFERENCES}

Abouelhoda, M., Issa, S. A., and Ghanem, M. (2012). Tavaxy: Integrating Taverna and Galaxy workflows with cloud computing support. BMC bioinformatics, 13(1):77.

Albert, R. (2005). Scale-free networks in cell biology. J Cell Sci, 118(Pt 21):4947-4957.

Alizadeh, A. A., Eisen, M. B., Davis, R. E., Ma, C., Lossos, I. S., Rosenwald, A., Boldrick, J. C., Sabet, H., Tran, T., Yu, X., Powell, J. I., Yang, L., Marti, G. E., Moore, T., Hudson, J., Lu, L., Lewis, D. B., Tibshirani, R., Sherlock, G., Chan, W. C., Greiner, T. C., Weisenburger, D. D., Armitage, J. O., Warnke, R., Levy, R., Wilson, W., Grever, M. R., Byrd, J. C., Botstein, D., Brown, P. O., and Staudt, L. M. (2000). Distinct types of diffuse large B-cell lymphoma identified by gene expression profiling. Nature, 403(6769):503-11.

Ashburner, M., Ball, C. A., Blake, J. A., Botstein, D., Butler, H., Cherry, J. M., Davis, A. P., Dolinski, K., Dwight, S. S., Eppig, J. T., Harris, M. A., Hill, D. P., Issel-Tarver, L., Kasarskis, A., Lewis, S., Matese, J. C., Richardson, J. E., Ringwald, M., Rubin, G. M., Sherlock, G., and Consortium, G. O. (2000). Gene Ontology: tool for the unification of biology. Nature Genetics, 25(1):25-29.

Balaur, I., Saqi, M., Barat, A., Lysenko, A., Mazein, A., Rawlings, C. J., Ruskin, H. J., and Auffray, C. (2016). EpiGeNet: A Graph Database of Interdependencies Between Genetic and Epigenetic Events in Colorectal Cancer. Journal of Computational Biology, 23(0):cmb.2016.0095.

Barabasi, A.-L. (2009). Scale-Free Networks: A Decade and Beyond. Science, 325(5939):412-413.

Barrett, T., Wilhite, S. E., Ledoux, P., Evangelista, C., Kim, I. F., Tomashevsky, M., Marshall, K. A., Phillippy, K. H., Sherman, P. M., Holko, M., Yefanov, A., Lee, H., Zhang, N., Robertson, C. L., Serova, N., Davis, S., and Soboleva, A. (2013). NCBI GEO: Archive for functional genomics data sets - Update. Nucleic Acids Research, 41(D1):991-5.

Bastian, M., Heymann, S., and Jacomy, M. (2009). Gephi : An Open Source Software for Exploring and Manipulating Networks Visualization and Exploration of Large Graphs. ICWSM.

Belmann, P., Dröge, J., Bremges, A., McHardy, A. C., Sczyrba, A., and Barton, M. D. (2015). Bioboxes: standardised containers for interchangeable bioinformatics software. GigaScience, 4(1):47.

Boettiger, C. (2015). An introduction to Docker for reproducible research. ACM SIGOPS Operating Systems Review, 49(1):71-79.

Carata, L., Akoush, S., Balakrishnan, N., Bytheway, T., Sohan, R., Seltzer, M., and Hopper, A. (2014). A Primer on Provenance. Communications of the ACM, 57(5):52-60.

Choobdar, S., Ribeiro, P., and Silva, F. (2015). Discovering weighted motifs in gene co-expression networks. Proceedings of the 30th Annual ACM Symposium on Applied Computing - SAC'15, pages 10-17.

Conesa, A., Madrigal, P., Tarazona, S., Gomez-Cabrero, D., Cervera, A., McPherson, A., Szcześniak, M. W., Gaffney, D. J., Elo, L. L., Zhang, X., and Mortazavi, A. (2016). A survey of best practices for RNA-seq data analysis. Genome Biology, 17(1):13. 
Crick, F. (1970). Central Dogma of Molecular Biology. Nature, 227(5258):561-563.

Daniels, J. (2009). Server virtualization architecture and implementation. Crossroads, 16(1):8-12.

de Paula, R., Holanda, M., Gomes, L. S. A., Lifschitz, S., and Walter, M. E. M. T. (2013). Provenance in bioinformatics workflows. BMC bioinformatics, 14 Suppl 1(Suppl 11):S6.

Deelman, E., Gannon, D., Shields, M., and Taylor, I. (2009). Workflows and e-Science: An overview of workflow system features and capabilities. Future Generation Computer Systems, 25(5):528-540.

Di Tommaso, P., Palumbo, E., Chatzou, M., Prieto, P., Heuer, M. L., and Notredame, C. (2015). The impact of Docker containers on the performance of genomic pipelines. PeerJ, 3:e1273.

Dudoit, S., Gentleman, R. C., and Quackenbush, J. (2003). Open source software for the analysis of microarray data. BioTechniques, 34(3 SUPPL.):45-51.

Ewald, D. A., Malajian, D., Krueger, J. G., Workman, C. T., Wang, T., Tian, S., Litman, T., Guttman-Yassky, E., and Suárez-Fariñas, M. (2015). Meta-analysis derived atopic dermatitis (MADAD) transcriptome defines a robust AD signature highlighting the involvement of atherosclerosis and lipid metabolism pathways. BMC medical genomics, 8:60.

Falcon, S. and Gentleman, R. (2007). Using GOstats to test gene lists for GO term association. Bioinformatics, 23(2):257-258.

Falcon, S., Morgan, M., and Gentleman, R. (2007). An Introduction to Bioconductor's ExpressionSet Class. Technical report.

Franceschini, A., Szklarczyk, D., Frankild, S., Kuhn, M., Simonovic, M., Roth, A., Lin, J., Minguez, P., Bork, P., Von Mering, C., and Jensen, L. J. (2013). STRING v9.1: Protein-protein interaction networks, with increased coverage and integration. Nucleic Acids Research, 41(D1):808-815.

Gadelha, L. M. R., Wilde, M., Mattoso, M., and Foster, I. (2012). MTCProv: A practical provenance query framework for many-task scientific computing. Distributed and Parallel Databases, 30(5-6):351-370.

Garcia-Molina, H., Ullman, J., and Widom, J. (2009). Database systems: the complete book.

Gautier, L., Cope, L., Bolstad, B. M., and Irizarry, R. A. (2004). Affy - Analysis of Affymetrix GeneChip data at the probe level. Bioinformatics, 20(3):307-315.

Ghosh, S., Matsuoka, Y., Asai, Y., Hsin, K.-Y., and Kitano, H. (2011). Software for systems biology: from tools to integrated platforms. Nature Reviews Genetics, 12(12):821-832.

Giardine, B., Riemer, C., Hardison, R. C., Burhans, R., Elnitski, L., Shah, P., Zhang, Y., Blankenberg, D., Albert, I., Taylor, J., Miller, W., Kent, W. J., and Nekrutenko, A. (2005). Galaxy: A platform for interactive large-scale genome analysis. Genome Research, 15(10):1451-1455.

Goble, C. A. and De Roure, D. C. (2007). myExperiment. Proceedings of the 2nd workshop on Workflows in support of large-scale science - WORKS '07, pages 1-2.

Golub, T. R., Slonim, D. K., Tamayo, P., Huard, C., Gaasenbeek, M., Mesirov, J. P., Coller, H., Loh, M. L., Downing, J. R., Caligiuri, M. A., Bloomfield, C. D., and Lander, E. S. (1999). Molecular classification of cancer: class discovery and class prediction by gene expression monitoring. Science, 286(5439):531-537.

Guinney, J., Dienstmann, R., Wang, X., de Reyniès, A., Schlicker, A., Soneson, C., Marisa, L., Roepman, P., Nyamundanda, G., Angelino, P., Bot, B. M., Morris, J. S., Simon, I. M., Gerster, S., Fessler, E., De Sousa E Melo, F., Missiaglia, E., Ramay, H., Barras, D., Homicsko, K., Maru, D., Manyam, G. C., Broom, B., Boige, V., Perez-Villamil, B., Laderas, T., Salazar, R., Gray, J. W., Hanahan, D., Tabernero, J., Bernards, R., Friend, S. H., Laurent-Puig, P., Medema, J. P., Sadanandam, A., Wessels, L., Delorenzi, M., Kopetz, S., Vermeulen, L., and Tejpar, S. (2015). The consensus molecular subtypes of colorectal cancer. Nature Medicine, 21(11):1350-1356.

Gundersen, G. W., Jagodnik, K. M., Woodland, H., Fernandez, N. F., Sani, K., Dohlman, A. B., Ung, P. M.-U., Monteiro, C. D., Schlessinger, A., and Ma'ayan, A. (2016). GEN3VA: aggregation and analysis of gene expression signatures from related studies. BMC Bioinformatics, 17(1):461.

Have, C. T., Jensen, L. J., and Wren, J. (2013). Are graph databases ready for bioinformatics? Bioinformatics, 29(24):3107-3108

Henkel, R., Wolkenhauer, O., and Waltemath, D. (2015). Combining computational models, semantic annotations and simulation experiments in a graph database. Database, 2015:1-16.

Henry, V. J., Bandrowski, A. E., Pepin, A. S., Gonzalez, B. J., and Desfeux, A. (2014). OMICtools: an informative directory for multi-omic data analysis. Database : the journal of biological databases and curation, 2014(0):bau069bau069.

Hosny, A., Vera-Licona, P., Laubenbacher, R., and Favre, T. (2016). AlgoRun: a Docker-based packaging system for platform-agnostic implemented algorithms. Bioinformatics (Oxford, England), 32(15):2396-8. 
Hung, L. H., Kristiyanto, D., Lee, S. B., and Yeung, K. Y. (2016). GUIdock: Using Docker containers with a common graphics user interface to address the reproducibility of research. PLOS ONE, 11(4):e0152686.

Irizarry, R. A., Hobbs, B., Collin, F., Beazer-Barclay, Y. D., Antonellis, K. J., Scherf, U., and Speed, T. P. (2003). Exploration, normalization, and summaries of high density oligonucleotide array probe level data. Biostatistics, 4(2):249-264.

Johnson, D., Johnson, D., Connor, A., McKeever, S., Wang, Z., Deisboeck, T., Quaiser, T., and Shochat, E. (2014). Semantically Linking In Silico Cancer Models. Cancer Informatics, 13(Suppl 1):133.

Joyce, A. R. and Palsson, B. O. (2006). The model organism as a system: integrating 'omics' data sets. Nature reviews. Molecular cell biology, 7(3):198-210.

Kendall, M. G. and Bradford Hill, A. (1953). Royal Statistical Society. Nature, 116(1):11-34.

Langfelder, P. and Horvath, S. (2008). WGCNA: an R package for weighted correlation network analysis. BMC bioinformatics, 9:559.

Lerner, B. S. and Boose, E. R. (2015). RDataTracker and DDG Explorer. In Revised Selected Papers of the 5th International Provenance and Annotation Workshop on Provenance and Annotation of Data and Processes - Volume 8628, pages 288-290. Springer-Verlag New York, Inc.

Liu, J., Pacitti, E., Valduriez, P., and Mattoso, M. (2015). A Survey of Data-Intensive Scientific Workflow Management. Journal of Grid Computing, 13(4):457-493.

Lohse, M., Bolger, A. M., Nagel, A., Fernie, A. R., Lunn, J. E., Stitt, M., and Usadel, B. (2012). RobiNA: A user-friendly, integrated software solution for RNA-Seq-based transcriptomics. Nucleic Acids Research, 40(W1):622-627.

Lysenko, A., Roznovăț, I. A., Saqi, M., Mazein, A., Rawlings, C. J., Auffray, C., Auffray, C., Charron, D., Hood, L., Hood, L., Tian, Q., Callahan, A., Cruz-Toledo, J., Ansell, P., Dumontier, M., Köhler, J., Baumbach, J., Taubert, J., Lysenko, A., Lysenko, A., Hindle, M., Eronen, L., Toivonen, H., Tatusova, T., Ciufo, S., Fedorov, B., Sayers, E., Barrett, T., Benson, D., Bairoch, A., Kerrien, S., Aranda, B., Breuza, L., Croft, D., Mundo, A., Haw, R., BauerMehren, A., Bundschus, M., Rautschka, M., Knox, C., Law, V., Jewison, T., Uhlén, M., Fagerberg, L., Hallström, B., Kaneko, Y., Yatagai, Y., Yamada, H., Voraphani, N., Gladwin, M., Contreras, A., Modena, B., Tedrow, J., Milosevic, J., Durrington, H., Farrow, S., Loudon, A., Ray, D., Ko, C., Takahashi, J., Lesk, V., Taubert, J., Rawlings, C., Sternberg, M., Tamaddoni-Nezhad, A., Lesk, V., Côté, R., Rogers, F., Ashburner, M., Ball, C., Blake, J., Brinkman, R., Courtot, M., Derom, D., Smith, B., Ashburner, M., Rosse, C., Lassila, O., Swick, R., Wide, W., and Consortium, W. (2016). Representing and querying disease networks using graph databases. BioData Mining, 9(1):23.

Madhamshettiwar, P. B., Maetschke, S. R., Davis, M. J., and Ragan, M. a. (2013). RMaNI: Regulatory Module Network Inference framework. BMC bioinformatics, 14 Suppl 1(Suppl 16):S14.

Mathelier, A., Zhao, X., Zhang, A. W., Parcy, F., Worsley-Hunt, R., Arenillas, D. J., Buchman, S., Chen, C. Y., Chou, A., Ienasescu, H., Lim, J., Shyr, C., Tan, G., Zhou, M., Lenhard, B., Sandelin, A., and Wasserman, W. W. (2014). JASPAR 2014: An extensively expanded and updated open-access database of transcription factor binding profiles. Nucleic Acids Research, 42(D1):1-6.

Maule, A., Emmerich, W., and Rosenblum, D. (2008). Impact analysis of database schema changes. 2008 ACM/IEEE 30th International Conference on Software Engineering, pages 451-460.

Medina, I., Carbonell, J., Pulido, L., Madeira, S. C., Goetz, S., Conesa, A., Tárraga, J., Pascual-Montano, A., NogalesCadenas, R., Santoyo, J., García, F., Marbà, M., Montaner, D., and Dopazo, J. (2010). Babelomics: An integrative platform for the analysis of transcriptomics, proteomics and genomic data with advanced functional profiling. Nucleic Acids Research, 38(SUPPL. 2):210-213.

Merkel, D. (2014). Docker: lightweight Linux containers for consistent development and deployment. Linux Journal, 2014(239):76-91.

Murta, L., Braganholo, V., Chirigati, F., Koop, D., and Freire, J. (2015). No Workflow: Capturing and analyzing provenance of scripts. In Lecture Notes in Computer Science (including subseries Lecture Notes in Artificial Intelligence and Lecture Notes in Bioinformatics), volume 8628, pages 71-83. Springer, Cham.

Muth, T., Behne, A., Heyer, R., Kohrs, F., Benndorf, D., Hoffmann, M., Lehtevä, M., Reichl, U., Martens, L., and Rapp, E. (2015). The MetaProteomeAnalyzer: A powerful open-source software suite for metaproteomics data analysis and interpretation. Journal of Proteome Research, 14(3):1557-1565.

Oinn, T., Addis, M., Ferris, J., Marvin, D., Senger, M., Greenwood, M., Carver, T., Glover, K., Pocock, M. R., Wipat, A., and Li, P. (2004). Taverna: A tool for the composition and enactment of bioinformatics workflows. Bioinformatics, 20(17):3045-3054.

Preusse, M., Theis, F. J., and Mueller, N. S. (2016). miTALOS v2: Analyzing tissue specific microRNA function. PLoS ONE, 11(3):1-15. 
Reynolds, A. P., Richards, G., De La Iglesia, B., and Rayward-Smith, V. J. (2006). Clustering rules: A comparison of partitioning and hierarchical clustering algorithms. Journal of Mathematical Modelling and Algorithms, 5(4):475504.

Robles, A. I. and Harris, C. C. (2016). Integration of multiple "OMIC" biomarkers: A precision medicine strategy for lung cancer. Lung Cancer.

Sandve, G. K., Nekrutenko, A., Taylor, J., and Hovig, E. (2013). Ten Simple Rules for Reproducible Computational Research. PLoS Computational Biology, 9(10):1-4.

Schuler, G. D., Epstein, J. A., Ohkawa, H., and Kans, J. A. (1996). [10] Entrez: Molecular biology database and retrieval system. Methods in Enzymology, 266:141-162.

Schulze, K., Imbeaud, S., Letouzé, E., Alexandrov, L. B., Calderaro, J., Rebouissou, S., Couchy, G., Meiller, C., Shinde, J., Soysouvanh, F., Calatayud, A.-L., Pinyol, R., Pelletier, L., Balabaud, C., Laurent, A., Blanc, J.-F., Mazzaferro, V., Calvo, F., Villanueva, A., Nault, J.-C., Bioulac-Sage, P., Stratton, M. R., Llovet, J. M., and Zucman-Rossi, J. (2015). Exome sequencing of hepatocellular carcinomas identifies new mutational signatures and potential therapeutic targets. Nature Genetics, 47(5):505-511.

Shade, A. and Teal, T. K. (2015). Computing Workflows for Biologists: A Roadmap. PLoS Biology, 13(11):e1002303. Siegel, R. L., Miller, K. D., and Jemal, A. (2017). Cancer statistics, 2017. CA: A Cancer Journal for Clinicians, 67(1):7-30.

Smoot, M. E., Ono, K., Ruscheinski, J., Wang, P. L., and Ideker, T. (2011). Cytoscape 2.8: New features for data integration and network visualization. Bioinformatics, 27(3):431-432.

Smyth, G. K. (2004). Linear Models and Empirical Bayes Methods for Assessing Differential Expression in Microarray Experiments Linear Models and Empirical Bayes Methods for Assessing Differential Expression in Microarray Experiments. Statistical Applications in Genetics and Molecular Biology, 3(1):1-26.

Stonebraker, M. (2010). SQL databases v. NoSQL databases. Communications of the ACM, 53(4):10.

Szitenberg, A., John, M., Blaxter, M. L., and Lunt, D. H. (2015). ReproPhylo: An Environment for Reproducible Phylogenomics. PLoS Computational Biology, 11(9):1-13.

Timmons, J. A., Szkop, K. J., and Gallagher, I. J. (2015). Multiple sources of bias confound functional enrichment analysis of global -omics data. Genome Biology, 16(1):186.

Ulitsky, I., Maron-Katz, A., Shavit, S., Sagir, D., Linhart, C., Elkon, R., Tanay, A., Sharan, R., Shiloh, Y., and Shamir, R. (2010). Expander: from expression microarrays to networks and functions. Nature protocols, 5(2):303-22.

Wang, Y.-H., Cheng, T.-Y., Chen, T.-Y., Chang, K.-M., Chuang, V. P., and Kao, K.-J. (2014). Plasmalemmal Vesicle Associated Protein (PLVAP) as a therapeutic target for treatment of hepatocellular carcinoma. BMC cancer, 14(1):815.

Wilde, M., Hategan, M., Wozniak, J. M., Clifford, B., Katz, D. S., and Foster, I. (2011). Swift: A language for distributed parallel scripting. Parallel Computing, 37(9):633-652.

Wilson, G., Aruliah, D. A., Brown, C. T., Chue Hong, N. P., Davis, M., Guy, R. T., Haddock, S. H. D., Huff, K. D., Mitchell, I. M., Plumbley, M. D., Waugh, B., White, E. P., and Wilson, P. (2014). Best Practices for Scientific Computing. PLoS Biology, 12(1):e1001745.

Zhang, B. and Horvath, S. (2005). A general framework for weighted gene co-expression network analysis. Statistical applications in genetics and molecular biology, 4(1):Article17.

Zhang, H. M., Chen, H., Liu, W., Liu, H., Gong, J., Wang, H., and Guo, A. Y. (2012a). AnimalTFDB: A comprehensive animal transcription factor database. Nucleic Acids Research, 40(D1):144-149.

Zhang, J., Lu, K., Xiang, Y., Islam, M., Kotian, S., Kais, Z., Lee, C., Arora, M., Liu, H. w., Parvin, J. D., and Huang, K. (2012b). Weighted Frequent Gene Co-expression Network Mining to Identify Genes Involved in Genome Stability. PLoS Computational Biology, 8(8).

Zhao, S., Fung-Leung, W. P., Bittner, A., Ngo, K., and Liu, X. (2014). Comparison of RNA-Seq and microarray in transcriptome profiling of activated T cells. PLoS ONE, 9(1):e78644.

Zichen, W., Ma'ayn, A., Wang, Z., and Ma'ayan, A. (2016). An open RNA-Seq data analysis pipeline tutorial with an example of reprocessing data from a recent Zika virus study. F1000Research, 5:1574. 